\title{
Neue gesellschaftliche Herausforderungen im Naturgefahrenmanagement: Welche Entwicklungen kommen auf uns zu?
}

\author{
Thomas Thaler · Marie-Sophie Attems · Sven Fuchs
}

Online publiziert: 22. Januar 2018

(c) Der/die Autor(en) 2018. Dieser Artikel ist eine Open-Access-Publikation.

\begin{abstract}
Zusammenfassung In Österreich sind in den vergangenen Jahren wiederholt Naturgefahren aufgetreten, die in der Politik, aber auch in der Bevölkerung verstärkt durch die Meldungen in den

New societal challenges for transformation in natural hazard management: adaptation strategies necessary to manage future developments
\end{abstract} Medien - immer wieder als Jahrhundertereignisse bezeichnet wurden. Die hohen volkswirtschaftlichen und privaten Schäden stellen die Frage in den Vordergrund, welche gesellschaftlichen Anpassungsstrategien diese Ereignisse im Lichte des Klimawandels erfordern. Speziell die ökonomischen Verluste und sozialen Beeinträchtigungen durch hydrologische Extremereignisse haben in den letzten Jahren gezeigt, dass traditionelle Schutzstrategien an ihre Grenzen stoßen. Im Forschungsprojekt TRANSADAPT untersucht das Institut für Alpine Naturgefahren der Universität für Bodenkultur Wien gemeinsam mit Partnern aus verschiedenen europäischen Ländern innovative Lösungen, um besser auf zukünftige Extremereignisse vorbereitet zu sein. Diese vereinen traditionelle bauliche Schutzstrategien mit alternativen Nutzungskonzepten von Schutzsystemen, und umfassen beispielsweise die Freizeitnutzung von Überschwemmungsflächen, die Integration von Schutzdämmen in städtebauliche Nutzungen oder Mehrfachnutzungen von Dämmen durch die Integration von kulturell nutzbarem Raum sowie Einkaufszentren.

Schlüsselwörter Risiko Governance . Formative Szenarioanalyse . Naturgefahrenmanagement · Multiple Nutzung

MMag. T. Thaler, $\mathrm{PhD}(\varangle)$.

M.-S. Attems, MSc · PD Dr. S. Fuchs Institut für Alpine Naturgefahren, Universität für Bodenkultur Wien, Peter-Jordan-Straße 82, 1190 Wien, Österreich

thomas.thaler@boku.ac.at
Abstract Losses from extreme hydrological events, such as recently experienced in Europe have focused the attention of policymakers as well as researchers on vulnerability to natural hazards in response to increasing flood risks, individual citizens and local communities are setting-up initiatives for flood risk management that may complement more top-down initiatives by national governments. A major challenge is to represent the complexity of coupled human-environmental systems and particularly the feedback loops between environmental dynamics and human decision-making processes on different scales. The JPIfunded project TRANS-ADAPT focuses on initiatives to flood risk management which are different from the mainstream. Through such distance from the mainstream, community initiatives may facilitate change that is complementary or disruptive to embedded policies. These initiatives are often pursued by local actors and stakeholders, not with the aim of contributing to broader societal transformation but to address local problems or to seize local opportunities.

Keywords Risk governance $\cdot$ Formative scenario analysis - Natural hazard management $\cdot$ Multiple-use

\section{Einleitung}

Naturgefahren spielen im 21. Jahrhundert eine bedeutsame Rolle - vor allem, wenn die Gesellschaft durch Extremereignisse betroffen ist. Wiederholt treten Ereignisse auf, die hohe Schäden für die Gesellschaft bedeuten, und eine dementsprechende Beeinträchtigung mit sich bringen. Die Herausforderung, eine adäquate Strategie gegenüber Extremereignissen $\mathrm{zu}$ haben, wird unter anderem durch die nachweisbar anhaltenden Bauaktivitäten in manchen Gefährdungsbereichen erschwert. Häufig werden Wohn- bzw. Industriegebäude oder andere Infrastrukturprojekte wie beispielsweise Straßen in gefährdeten Bereichen genehmigt und errichtet. Gründe hierfür sind einerseits im Aspekt kostengünstiger Grundstücke zu suchen, und andererseits mancherorts Flächen im Überschwemmungsbereich, die die letzten Entwicklungsmöglichkeiten für eine Gemeinde oder Region darstellen, da sonst sämtliche Entwicklungspotenziale bereits ausgeschöpft sind (White 1945; Burton und Kates 1964; White et al. 2001; Fuchs et al. 2015, 2017). In alpinen Talräumen Österreichs, Italiens oder der Schweiz sind zudem topografische Einschränkungen gegeben, und die geringen als Dauersiedlungsraum zur Verfügung stehenden Flächen stellen eine große politische Herausforderung dar, um einerseits lokale oder regionale sozioökonomische Entwicklung zu ermöglichen und andererseits einen Schutz vor zukünftigen Naturgefahrenereignissen zu gewähren (Fuchs et al. 2015, 2017).

Diese Dichotomie zwischen lokaler Entwicklung und dem Unterbinden von höherer Exposition von Risikoobjekten (beispielsweise über Bauverbote) sind durch die extremen Hochwässer der vergangenen Jahre immer stärker in den Fokus der Politik und der Öffentlichkeit gerückt, unter anderem aufgrund von Fragestellungen nach der Haftung im Schadensfall, aber auch in Hinblick auf notwendige politische Entscheidungen, die mittelfristig alternative Maßnahmen im Vergleich zu den traditionellen Schutzbauwerken erfordern können. Die Folge ist, dass insbesondere in Gemeinden ohne geeignete Entwicklungsgebiete ein extremer Nutzungsdruck entsteht, und 
diese im Spannungsfeld unterschiedlicher Landnutzungsansprüche stehen (Thaler 2014; Steinhäußer et al. 2015).

Alternative Schutzmaßnahmen erfordern ein Umdenken im Naturgefahrenmanagement, es stellen sich insbesondere Fragen nach der Rolle bzw. Aufgabe der öffentlichen Hand und privater Akteure, wie beispielsweise in Bezug auf Fragen der individuellen Vorsorge oder der Einbindung der Bevölkerung in den Planungsprozess (Holub und Fuchs 2009; Hartmann 2011; Adger et al. 2013; Thaler und Levin-Keitel 2016). Es stellt sich zum Beispiel bereits bei der retentionswirksamen Bewirtschaftung eines Einzugsgebietes die Frage, ob diese Maßnahme innerhalb einer Gemeinde oder interkommunal durchgeführt werden soll, um den größtmöglichen Nutzen für alle Beteiligten $\mathrm{zu}$ erzielen. Hierbei geht es auch um Fragen der Motivation, da sich Naturgefahren nicht an politischen Grenzen orientieren. Eine mögliche Folge ungeklärter Rollenverteilung ist, dass eine Gemeinde die Nutzung von Flächen für Schutzkonzepte innerhalb ihres Hoheitsgebietes einschränken muss, was wiederum $\mathrm{zu}$ zahlreichen weiteren Konflikten führen kann (Hartmann 2011; Thaler et al. 2016). Die Möglichkeiten alternativer Rollenverteilung verändert die Interaktion mit der Bevölkerung, da lokale Bedürfnisse in der Planung und Umsetzung heutzutage verstärkt berücksichtigt werden müssen (Ayre und Nettle 2015; Thaler et al. 2016). Insbesondere in Zusammenhang mit der Option, Gefährdungsgebiete retentionswirksam $\mathrm{zu}$ bewirtschaften, ehemalige Überflutungs- und Ablagerungsflächen wiederherzustellen, oder auch gezielt $\mathrm{Ab}$ - oder Umsiedelungen und Umwidmungen von Siedlungsflächen vorzunehmen wie beispielsweise in Machland-Nord oder aktuell im Eferdinger Becken in Oberösterreich -, ist eine verstärkte Zusammenarbeit zwischen lokaler Politik und Bevölkerung zur erfolgreichen Umsetzung dieser Maßnahmen notwendig (EU 2007; BMLFUW 2014).

Weitere Beispiele, die eine starke lokale Kooperation bedingen, stellen sogenannte multifunktionale Schutzkonzepte dar, wie die Freizeitnutzung von Überschwemmungsflächen, die Integration von Schutzmaßnahmen in städtebauliche Bedürfnisse und die Kombination von Schutzmaßnahmen mit kulturellen Einrichtungen oder Einkaufsflächen. Derartige Anpassungs- strategien erfordern einen Dialog zwischen allen beteiligten Akteuren, und insbesondere sind Gesellschaft und Politik gefordert, eine erforderliche Neuausrichtung der Verantwortlichkeit zwischen der öffentlichen Hand und der Gesellschaft zu definieren. Diese Neuausrichtung beinhaltet innovative Konzepte der (Ko-)Finanzierung von Schutzbauwerken und die Entwicklung erforderlicher Anpassungsstrategien, wie sie sich aus den Anforderungen des Klimawandels und den sozio-ökonomischen Entwicklungen ergeben (Fuchs 2009; Keiler et al. 2010). Die Umsetzung multifunktionaler Schutzkonzepte schafft häufig neue Handlungsfelder und -räume für die lokale Bevölkerung (Thaler et al. 2017) und eröffnet innovative Zugänge für beteiligte EntscheidungsträgerInnen.

Diese Neuausrichtung der Verantwortlichkeit kann aber gleichzeitig zu neuen Konflikten führen; diese beinhalten die Frage, welche Flächen für das Schutzkonzept verwendet werden und welche Doppelfunktionen umgesetzt werden können, wie die Finanzierung erfolgt und wie die Instandhaltung geregelt ist. Hierbei ändert sich auch das Anforderungsprofil an die öffentliche Hand: Je integrativer eine Planung von Schutzkonzepten wird, desto stärker muss die öffentliche Hand Aufgaben im Bereich des Projekt- und Konfliktmanagements übernehmen (Thaler und Levin-Keitel 2016). Zusätzlich sehen wir eine Veränderung im Bereich der lokalen politischen Ebene, da sich die Umsetzung multifunktionaler Schutzkonzepte meist außerhalb des traditionellen politischen Rahmens präsentiert. Der Aufbau sowie die Nutzung informeller Netzwerke und Kooperationen sind zentrale Stützpfeiler einer solchen Neuausrichtung des Naturgefahrenmanagements, um überhaupt multifunktionale Schutzkonzepte zu ermöglichen (Allmendinger und Haughton 2012; Thaler 2016). Informelle Interaktionen haben den Vorteil, dass neue kreative Lösungsvorschläge außerhalb des klassischen politischen Planungsprozesses ermöglicht und gefördert werden, wobei diese mit einem entsprechend hohen $\mathrm{Maß}$ an lokaler Bevölkerungsbeteiligung erfolgen müssen. Das Denken und Handeln außerhalb der traditionellen politischen Grenzen ermöglicht erfahrungsgemäß überhaupt erst die Entstehung neuer innovativer Lösungen (Sielker 2016). Darüber hinaus gestatten diese informellen Kooperatio- nen häufig eine erhöhte Flexibilität im Planungsprozess, der wiederum insbesondere bei der Umsetzung multifunktionaler Schutzkonzepte essenziell ist. Der Konflikt, der zwischen den informellen Kooperationen und den formellen Instrumenten besteht, bedeutet allerdings eine Herausforderung für alle beteiligten Akteure (Allmendinger und Haughton 2009, 2010; Metzger und Schmitt 2012; Haughton et al. 2013).

Im Forschungsprojekt TRANSADAPT untersuchen WissenschaftlerInnen der Universität für Bodenkultur Wien gemeinsam mit Partnern aus weiteren europäischen Ländern innovative Lösungen, um zukünftig besser auf Extremereignisse vorbereitet zu sein. Diese Lösungen vereinen traditionelle bauliche Schutzstrategien vor Naturgefahren mit alternativen Nutzungskonzepten von Schutzsystemen. Beispiele umfassen u. a. die Freizeitnutzung von Überschwemmungsflächen oder die Integration von Einkaufszentren in Schutzmaßnahmen. Es wurden insgesamt acht Fallstudien aus vier europäischen Ländern ausgewählt, in denen ein multifunktionaler Nutzen der Schutzkonzepte umgesetzt wurde sowie zwei Beispiele, bei denen ein multifunktionales Schutzkonzept geplant wurde, das jedoch am Widerstand der Bevölkerung scheiterte und nicht umgesetzt werden konnte. Bei sämtlichen ausgewählten Beispielen war die lokale Bevölkerung die treibende Kraft. Einzelne Personen oder Personengruppen nutzten die Gunst der Stunde unmittelbar nach einem Ereignis (das sogenannte policy window), um ihre Interessen gegenüber der öffentlichen Hand vorzubringen. Die Hauptbeweggründe für das Engagement der Bevölkerung waren einerseits die aktuellen gesellschaftlichen Herausforderungen, wie z.B. Verringerung des Budgets für Naturgefahrenmanagement oder wie man mit zukünftigen Herausforderungen, wie z.B. dem Klimawandel umgehen kann.

Der folgende Beitrag beschäftigt sich mit der systemischen Betrachtungsweise der Umsetzung multifunktionaler Schutzkonzepte. Dafür wurde im Rahmen des Projekts ein transdisziplinärer Ansatz mittels der formativen Szenarioanalyse verwendet, um zu beantworten, wie multifunktionale Schutzkonzepte in den jeweiligen Ländern national umgesetzt werden können. Die Fallbeispiele aus Irland, Frankreich, den Niederlanden und Österreich sollten dabei die 
Zweckmäßigkeit und Herausforderungen einer Umsetzung multifunktionaler Schutzkonzepte im Naturgefahrenmanagement verdeutlichen. In diesem Beitrag wird insbesondere auf die Ergebnisse der österreichischen Fallstudien eingegangen. Es wird erörtert, welche Faktoren multifunktionelle Schutzkonzepte in Österreich beeinflussen und wie der institutionelle Rahmen dazu gestaltet sein muss.

\section{Methodische Herangehensweise}

Für den folgenden Beitrag wurde ein explorativer Szenarioanalyseansatz ausgewählt, um zu erörtern, wie wir lokale und innovative multifunktionale Schutzkonzepte auf nationaler Ebene umsetzen können. Die Szenarioanalyse ermöglicht es, zukünftige Entwicklungen im politischen Prozess abzubilden und zu analysieren. Dabei wird der aktuelle politische Prozess in einem vereinfachten Systembild dargestellt. Die formative Szenarioanalyse beinhaltet zwei Schwerpunkte: Der erste Abschnitt untersucht allgemein den Ist-Zustand des untersuchten Rahmens, der zweite Abschnitt beschäftigt sich schließlich mit der Zukunftsanalyse (Mazzorana et al. 2009). Die methodische Vorgehensweise berücksichtigt insgesamt 12 Schritte, die wiederum in fünf Phasen unterteilt werden können (Scholz und Tietje 2002; Spoerri et al. 2009). Diese fünf Phasen sind: (1) Systemdefinition und -analyse, (2) Einflussfaktoren, (3) Systemanalyse, (4) Konsistenzanalyse sowie (5) die Auswahl und Formulierung der möglichen Trendprojektionen.

\subsection{2 .1 Systemdefinition}

Der erste Schritt untersucht die aktuellen institutionellen Strukturen, administrative Abläufe sowie gesellschaftliche Entwicklungen und die Frage, wieso multifunktionale Schutzkonzepte in den verschiedenen europäischen Ländern umgesetzt wurden oder auch nicht. Es wird vor allem auf die Frage fokussiert, wann solche innovativen Lösungsansätze im Naturgefahrenmanagement möglich und sinnvoll sind. Hierfür ist es zentral, die zeitlichen, thematischen und räumlichen Entwicklungen in der Analyse zu berücksichtigen, um einen Gesamteindruck sowie ein Verständnis für das System zu erlangen (Schläpfer 2006).

\subsection{Einflussfaktoren}

Aufbauend auf die Systemdefinition folgt in einem zweiten Schritt die Ermittlung der Einflussfaktoren und deren Wechselbeziehungen. Es werden Fragen untersucht, welche gesellschaftlichen, ökonomischen und politischen Entwicklungen die zukünftige Umsetzung von multifunktionalen Schutzkonzepten unterstützen oder behindern. Die Einflussfaktoren wurden von ausgewählten Stakeholdern der österreichischen Bundeswasserbauverwaltung und des Forsttechnischen Dienstes für Wildbach- und Lawinenverbauung ausgewählt und bewertet. Für die Bewertung der Wechselwirkungen wurden drei Kategorien verwendet: kein Einfluss, geringer Einfluss und starker Einfluss (Scholz und Tietje 2002). Das Ziel der Analyse besteht in der Untersuchung der jeweiligen verschiedenen wechselseitigen Beeinflussung der Faktoren.

\subsection{Konsistenzanalyse}

Im dritten Schritt erfolgte die Konsistenzanalyse. Mithilfe dieses Verfahrens wird die Logik der möglichen Szenarien ermittelt. Das Ziel besteht im Erreichen einer Homogenität bzw. eines hohen Konsistenzmaßes für die möglichen Zukunftsbilder. Basierend auf der Einflussmatrix des zweiten Schritts werden sämtliche Einflussfaktoren mit zwei Ausprägungen ermittelt. Die Werteskala verläuft zwischen Inkonsistenz und starker Konsistenz. Das Ziel liegt in einer Berechnung des Konsistenzmaßes der möglichen Entwicklungen anhand statistischer Berechnungen sowie einer Ermittlung der Reihenfolge für die Auswahl der verschiedenen Möglichkeiten.

\subsection{Auswahl und Formulierung der möglichen Entwicklungen}

Der letzte Schritt in der formativen Szenarioanalyse sieht die Auswahl und die Formulierung der möglichen Entwicklungen anhand der Kombinationen unterschiedlicher Einflussfaktoren vor. Das Auswahlverfahren basiert auf der errechneten Reihenfolge der konsistentesten Möglichkeiten.

\section{Multifunktionale Schutzkonzepte} in Europa

\subsection{Einflussfaktoren}

Grundsätzlich ergaben die Workshops verschiedene zentrale Einflussfaktoren, die für eine Umsetzung multifunktionaler Schutzkonzepte von zentraler Bedeutung sind. Diese können wiederum in die Teilbereiche Politik, Umwelt und Gesellschaft unterschieden werden. In Österreich wurden insgesamt zehn Einflussfaktoren als zentral für eine mögliche Umsetzung multifunktionaler Schutzkonzepte angesehen (Tab. 1).

Die Teilnehmenden der Workshops ermittelten mithilfe von Matrizen die ausgewählten Variablen auf deren direkte und indirekte Wechselwirkungen. Die Entscheidung der Höhe des Einflusses der Variablen wurde anhand eines Gruppenentscheidungsprozesses ermittelt. Für die österreichischen Konsensmatrizen konnten dabei insgesamt 90 Wechselbeziehungen ausfindig gemacht werden. Die einzelnen Variablen wurden in vier Teilbereiche unterteilt: (1) aktive Variablen, anhand derer sämtliche Einflussfaktoren zusammengefasst werden, die einen überdurchschnittlichen Einfluss auf die anderen Einflussfaktoren ausüben; (2) passive Variablen sind jene Variablen, die überdurchschnittlich von den restlichen Einflussfaktoren beeinflusst werden; (3) ambivalente Variablen sind Einflussgrößen, die überdurchschnittlich beeinflussen, aber auch gleichzeitig überdurchschnittlich beeinflusst werden, sowie (4) puffernde Variablen, die allgemein jene Einflussgrößen darstellen, die unterdurchschnittlich in das System eingreifen, aber auch unterdurchschnittlich beeinflusst werden. Aus dem österreichischen Workshop sind vor allem die beiden Variablen Ressourcenverfügbarkeit und Lifestyle

Tab. 1 Einflussfaktoren in Österreich

\begin{tabular}{|l|l|}
\hline Teilbereiche & Einflussfaktoren \\
\hline Politik & Institutioneller Rahmen \\
& Entscheidungsprozess \\
& Risikotransfer \\
\hline & Finanzierung \\
\hline & Risikoakzeptanz \\
& Administrative Rahmen \\
& Risikokommunikation \\
\hline Gesellschaft & Technische Entwicklungen \\
& Lifestyle \\
\hline Umwelt & Ressourcenverfügbarkeit \\
\hline
\end{tabular}




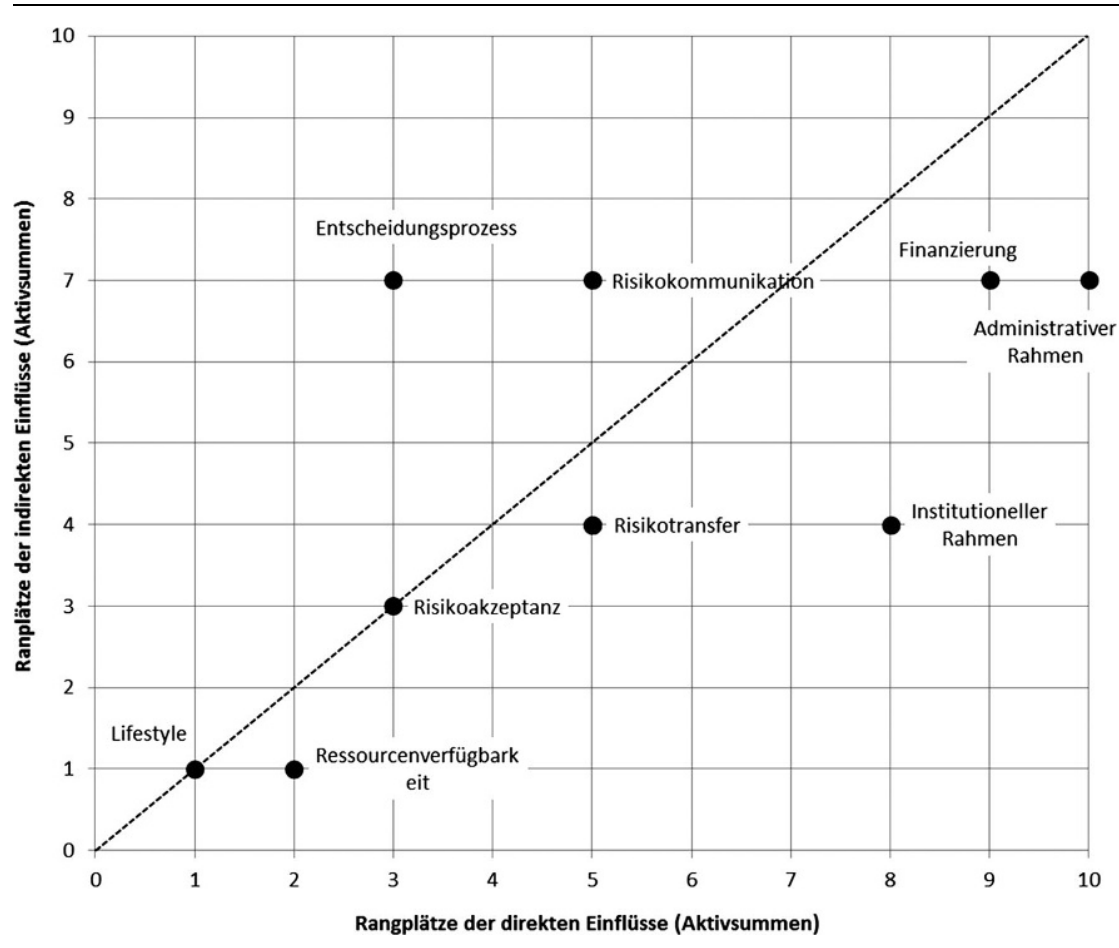

Abb. 1 Vergleich der direkten und indirekten Rangplätze der Einflussfaktoren

Schlüsselkonzepte in der erfolgreichen Umsetzung von multifunktionalen Schutzkonzepten. Speziell die Punkte Finanzierung (überraschenderweise), Risikokommunikation und administrativer Rahmen haben eine passive Rolle in der Umsetzung, d.h., diese Variablen spielen kaum eine Rolle.

Neben den direkten Wechselwirkungen spielt vor allem die indirekte Verbundenheit der Einflussfaktoren eine wesentliche Rolle im Entscheidungsprozess. Mithilfe einer sogenannten MIC-MAC-Analyse (Matrice d'Impacts Croisés - Multiplication Appliquée à un Classement) wurden die indirekten Wechselwirkungen der Einflussfakto- ren berechnet und evaluiert. Die MICMAC-Analyse stellt - ähnlich wie die Darstellung der direkten Einflussfaktoren - ein heuristisches Verfahren dar (Scholz und Tietje 2002). Werden die Ergebnisse betrachtet (direkte und indirekte Wechselwirkungen), sind - neben den bereits genannten aktiven Einflussfaktoren - weitere Variablen besonders relevant in der möglichen Umsetzung multifunktionaler Schutzkonzepte auf nationaler Ebene: die institutionellen Rahmenbedingungen sowie auch der technologische Fortschritt in einer Gesellschaft. Werden direkte und indirekte Einflussfaktoren verglichen wird deutlich, dass insbesondere der
Einflussfaktor der institutionellen Rahmenbedingungen unterbewertet wurde (siehe Abb. 1). Der institutionelle Rahmen stellt prinzipiell einen zentralen Punkt in der Umsetzung multifunktionaler Schutzkonzepte dar. Gibt es eine gesetzliche Einschränkung oder ein Verbot der Entwicklung und Umsetzung derartiger Schutzkonzepte (unter anderem wegen eines erhöhten Schadenspotenzials), würde dies bereits eine Planung und Umsetzung verhindern. Das bedeutet, dass der Gesetzgeber einen gesetzlichen Rahmen schaffen muss, um überhaupt multifunktionale Schutzkonzepte zu erlauben.

\subsection{Auswahl der Trendprojektionen}

In Tab. 2 sind zwei aus insgesamt 1024 möglichen sozio-politischen Entwicklungen im Zusammenhang mit multifunktionalen Schutzkonzepten in Österreich dargestellt. Die beiden ausgewählten Trendprojektionen zeigen jene Kombinationen, die aufbauend auf der computergestützten Analyse die konsistentesten Szenarien darstellen.

Das erste Entwicklungsszenario „Neuorientierung der Verantwortung“ zielt vorrangig auf die Herausforderung eines rechtlichen Rahmenwerkes mit der Frage nach der Verantwortlichkeit ab, wenn sich durch den Bau multifunktioneller Schutzbauwerke Personen verletzen würden. Das Szenario 1 verfolgt eine starke Kooperation zwischen der öffentlichen Hand (Behörde), der lokalen Politik sowie der Bevölkerung, um multifunktionale Schutzkonzepte erfolgreich umzusetzen. Die Kooperation sieht auch ein verstärktes Engagement zur Übernahme von Verantwortung auf der lokalen Ebene vor. Das Szenario 1 legt den Schwerpunkt insbesondere

Tab. 2 Überblick von potenziellen Entwicklungsszenarien

\begin{tabular}{l} 
Szenario 1: Neuorientierung der Verantwortung \\
Institutioneller Rahmen: Interpretationsspielraum aktiv nutzen, Regelung der \\
Verantwortung(sübernahme) \\
Entscheidungsprozess: konsensorientiert innerhalb der Gemeinde \\
Risikotransfer: Vorhanden \\
\hline Finanzierung: privates Interesse (Eigenfinanzierung) \\
Risikoakzeptanz: Abwägung der durch Maßnahme veränderten Risiken, Nut- \\
zen rechtfertigen zusätzliche Risiken \\
Administrative Rahmen: klassisches Verfahren \\
Risikokommunikation: Stärkung der Eigenverantwortung/Partizipation \\
Technische Entwicklungen: Stand der Technik ermöglicht Umsetzung einer \\
Maßnahme \\
Lifestyle: Wirtschaftliche Interessen/Rendite \\
Ressourcenverfügbarkeit: Hoher Nutzungsdruck \\
\hline
\end{tabular}

Szenario 2: Business as usual

Institutioneller Rahmen: Interpretationsspielraum aktiv nutzen, Regelung der Verantwortung(sübernahme)

Entscheidungsprozess: Einzelperson

Risikotransfer: nicht vorhanden

Finanzierung: erhöhtes öffentliches Interesse (Förderungen)

Risikoakzeptanz: im Verfahren Risikoaversion

Administrativer Rahmen: klassisches Verfahren

Risikokommunikation: Information

Technische Entwicklungen: Stand der Technik ermöglicht Umsetzung einer Maßnahme

Lifestyle: Wirtschaftliche Interessen/Rendite

Ressourcenverfügbarkeit: Hoher Nutzungsdruck 
auf die gemeinschaftliche Konsensentscheidung innerhalb einer Gemeinde, dies geschieht vor allem durch eine verstärkte Kooperation zwischen der öffentlichen Hand und der lokalen Bevölkerung. Diese Kooperation erfolgt über eine verstärkte Risikokommunikation und somit im Bereich der RisikoGovernance.

Das Szenario 2 „Business as usual" sieht einen verstärkten Top-downAnsatz bei der Entwicklung multifunktionaler Schutzkonzepte vor. Der Partizipationsprozess besteht hier vor allem aus einem Informationsaustausch statt einer starken Einbindung der lokalen Bevölkerung in die Politik der Planungs- und Umsetzungsprozesse. Der Entscheidungsprozess wird maßgeblich von einzelnen Akteuren getragen, aufgrund des dominanten Topdown-Ansatzes können hierbei jedoch kaum multifunktionale Schutzkonzepte entwickelt oder umgesetzt werden.

\section{Fazit und Ausblick}

Im Allgemeinen wird erwartet, dass es einerseits durch den Klimawandel, der in einigen Teilen Europas zu einer Erhöhung des Gefährdung durch Naturereignisse führt, und andererseits durch den sozio-ökonomischen Wandel zu neuen Anforderungen im Naturgefahrenmanagement kommen wird. Im Projekt TRANS-ADAPT wurde der Frage nach Folgewirkungen des aktuellen gesellschaftlichen Wandels auf das Naturgefahrenmanagement nachgegangen, insbesondere, wie innovative lokale Konzepte auf einer nationalen Ebene umgesetzt werden können und welche Faktoren diese Entwicklung beeinträchtigen. Die multifunktionalen Schutzkonzepte werden durch unterschiedliche Einflussfaktoren gesteuert und gefördert, wobei die Gemeinde bzw. die lokale Bevölkerung generell eine große Rolle für die Umsetzung spielen. Interessanterweise gibt es zahlreiche lokale Ideen und Konzepte, wie multifunktionale Schutzkonzepte aussehen können, und welche lokalen Bedürfnisse und Vorstellungen in den Planungsprozess von Schutzbauwerken eingebunden werden sollen. Die Beispiele gehen von einer Freizeitnut- zung hin zu kulturellen Einrichtungen oder Seminarräumlichkeiten, wodurch gleichzeitig eine verstärkte Risikokommunikation und die Schaffung von Risikobewusstsein ermöglicht werden.

Auf der anderen Seite gibt es auch Fallbeispiele, wo die Versuche einer Errichtung multifunktionaler Ansätze scheiterten. In diesen Fällen spielten einzelne Personengruppen eine zentrale Rolle in der Nichtumsetzung der Maßnahmen, wobei auch hier ein hoher Landnutzungsdruck sowie knappe finanzielle Ressourcen im Gemeindebudget vorherrschten.

Für eine erfolgreiche Umsetzung ist der hohe Nutzungsdruck ein wesentlicher Faktor in der Entwicklung innovativer Lösungen im Naturgefahrenmanagement. Das Ziel der multifunktionalen Schutzkonzepte besteht darin, dass der ungenutzte Raum in Schutzbauwerken optimal verwendet wird, wie beispielsweise in Großkirchheim mit dem Schießtunnel im Hochwasserdamm oder das Alpinarium in Galtür. Deshalb ist es nicht verwunderlich, dass vor allem in den oberen alpinen Einzugsgebieten ein solcher Bedarf weitaus größer ist als in jenen Gebieten, die keinen so deutlichen Landnutzungsdruck spüren. In diesem Zusammenhang spielen auch die jeweiligen lokalen Wirtschaftsinteressen eine große Rolle. Dieser Einflussfaktor korreliert häufig mit einem erhöhten Nutzungsdruck innerhalb der Gemeinde. Eine wesentliche Fragestellung besteht darin, wie in dem eingeschränkt zur Verfügung stehenden Raum sämtliche Interessen abgedeckt werden können. Einen weiteren Punkt stellen die institutionellen Bedingungen dar: Ohne die Ausnutzung rechtlich legaler Graubereiche bzw. der Möglichkeit, flexibel innerhalb des bestehenden Naturgefahrenmanagements $\mathrm{zu}$ agieren, können viele innovative Konzepte in Österreich und in anderen europäischen Ländern kaum durchgeführt werden. Eine zentrale Rolle spielte in allen Fallstudien die Frage der Haftung im Schadensfall. Weniger bedeutende Faktoren sind das Möglichkeitsfenster (policy window) für die Umsetzung multifunktionaler Schutzmaßnahmen sowie die Frage der Finanzierung derartiger Bauwerke. Ins- besondere wird die Möglichkeit des Aufbringens zusätzlicher finanzieller Mittel seitens privater Akteure (Public Private Partnership) bis jetzt kaum wahrgenommen.

Zusammenfassend ist eine erhöhte Partizipation der lokalen Politik und der Bevölkerung ein zentraler Schlüssel für die Planung und Umsetzung multifunktionaler Schutzkonzepte. Sobald für sämtliche beteilige Akteure ein Nutzen erkennbar wird, und sei es durch die Einbindung der lokalen Bevölkerung im Planungsprozess, wird der Nutzen derartiger Konzepte höher eingeschätzt. Dies kann nur durch eine entsprechende offene Einstellung der jeweiligen beteiligten Akteure geschehen. Dabei spielen Vertrauen und gegenseitige Anerkennung eine wichtige Rolle. Jene Beispiele, wo innovative Lösungsvorschläge scheiterten, zeigten eine unterschiedliche Auffassung des Planungsprozesses (soziale und kulturelle Unterschiede zwischen der öffentlichen und privaten Hand) sowie institutionelle Abhängigkeiten von technokratischen Ansätzen und Lösungen über die Bewertung von lokalen Lösungen.

Danksagung Der vorliegende Beitrag wurde im Rahmen des JPI-ClimateProjekts TRANS-ADAPT erarbeitet, die österreichischen Partner wurden durch das Bundesministerium für Wissenschaft, Forschung und Wirtschaft gefördert.

Funding Open access funding provided by University of Natural Resources and Life Sciences Vienna (BOKU).

Open Access Dieser Artikel wird unter der Creative Commons Namensnennung 4.0 International Lizenz (http:// creativecommons.org/licenses/by/4. 0/deed.de) veröffentlicht, welche die Nutzung, Vervielfältigung, Bearbeitung, Verbreitung und Wiedergabe in jeglichem Medium und Format erlaubt, sofern Sie den/die ursprünglichen Autor(en) und die Quelle ordnungsgemäß nennen, einen Link zur Creative Commons Lizenz beifügen und angeben, ob Änderungen vorgenommen wurden. 
Adger, N., Quinn, T., Lorenzoni, I., Murphy, C. \& Sweeney, J. (2013): Changing social contracts in climate-change adaptation. Nature Climate Change, 3, 330-333. https://doi.org/10.1038/ nclimatel751

Allmendinger, P. \& Haughton, G. (2009): Soft spaces, fuzzy boundaries, and metagovernance: The new spatial planning in the Thames Gateway. Environment and Planning A, 41, 617-633. https://doi.org/10.1068/a40208

Allmendinger, P. \& Haughton, G. (2010): Spatial planning, devolution, and new planning spaces. Environment and Planning C: Government and Policy, 28, 803-818. https://doi.org/10.1068/ c09163

Allmendinger, P. \& Haughton, G. (2012): Postpolitical spatial planning in England: A crisis of consensus? Transactions of the Institute of British Geographers, 37, 89-103. https://doi.org/10. 1111/j.1475-5661.2011.00468.

Ayre, M. \& Nettle, R. (2015): Doing integration in catchment management research: Insights into a dynamic learning process. Environmenta Science \& Policy, 47, 18-31. https://doi.org/10. 1016/j.envsci.2014.10.011

BMLFUW (2014): Maßnahmenkatalog des Hochwassermanagementplans. Wien: Bundesministerium für Land- und Forstwirtschaft, Um welt und Wasserwirtschaft

Burton, I. \& Kates, W. (1964): The perception of natural hazards in resource management. Natural Resources Journal, 3, 412-441.

EU (2007): Richtlinie 2007/60/EG des Europäischen Parlaments und des Rates vom 23. Oktober 2007 über die Bewertung und das Management von Hochwasserrisiken. Brüssel: Amtsblatt der Europäischen Union.

Fuchs, S. (2009): Susceptibility versus resilience to mountain hazards in Austria - Paradigm of vulnerability revisited. Natural Hazards and Earth System Sciences, 9, 337-352. https://doi org/10.5194/nhess-9-337-2009

Fuchs, S., Keiler, M. \& Zischg, A. (2015): A spatiotemporal multi-hazard exposure assessment based on property data. Natural Hazards and Earth System Sciences, 15, 2127-2142. https:// doi.org/10.5194/nhess-15-2127-2015

Fuchs, S., Röthlisberger, V., Thaler, T., Zischg

A. \& Keiler, M. (2017): Natural hazard manage- ment from a co-evolutionary perspective: exposure and policy response in the European Alps. Annals of the American Association of Geographers, 107, 382-392. https://doi.org/10.1080/ 24694452.2016.1235494

Hartmann, T. (2011): Clumsy floodplains: Responsive land policy for extreme floods. Farnham, Surrey: Ashgate

Haughton, G., Allmendinger, P. \& Oosterlynck, S. (2013): Spaces of neoliberal experimentation: Soft spaces, postpolitics, and neoliberal governmentality. Environment and Planning A, 45, 217-234. https://doi.org/10.1068/a45121

Holub, M. \& Fuchs, S. (2009): Mitigating mountain hazards in Austria - Legislation, risk transfer, and awareness building. Natural Hazards and Earth System Science, 9, 523-537. https:// doi.org/10.5194/nhess-9-523-2009

Keiler, M., Knight, J. \& Harrison, S. (2010): Climate change and geomorphological hazards in the eastern European Alps. Philosophical Trans actions of the Royal Society of London Series A, 368: 2461-2479. https://doi.org/10.1098/rsta. 2010.0047

Mazzorana, B., Hübl, J. \& Fuchs, S. (2009): Improving risk assessment by defining consistent and reliable system scenarios. Natural Hazards and Earth System Sciences, 9, 145-159. https:// doi.org/10.5194/nhess-9-145-2009

Metzger, J. \& Schmitt, P. (2012): When sof spaces harden: The EU strategy for the Baltic Sea Region. Environment and Planning A, 44, 263-280. https://doi.org/10.1068/a44188

Schläpfer, T. (2006): Einfluss der Hauptakteure im Lebenszyklus einer Immobilie. Masterarbeit ETH Zürich. Zürich: ETH Zürich.

Scholz, R. W. \& Tietje (2002): Embedded case study methods: Integrating quantitative and qualitative knowledge. Thousand Oaks, CA: Sage Publications

Sielker, F. (2016): New approaches in European governance? Perspectives of stakeholders in the Danube macroregion. Regional Studies Regional Science, 3, 88-95. https://doi.org/10.1080/ 21681376.2015.1116957

Spoerri, A., Lang, D. J., Binder, C. R. \& Scholz, R. (2009): Expert-based scenarios for strategic waste and resource management planning $C \& D$ waste recycling in the Canton of Zürich
Switzerland. Resources Conservation and Recycling, 53, 592-600. https://doi.org/10.1016/j. resconrec.2009.04.011

Steinhäußer, R., Siebert, R., Steinführer, A \& Hellmich, M. (2015): National and regiona land-use conflicts in Germany from the perspective of stakeholders. Land Use Policy, 49 183-194. https://doi.org/10.1016/j.landusepol. 2015.08.009

Thaler, T. (2014): Developing partnership approaches for flood risk management: Implementation of inter-local co-operations in Austria. Water International, 39, 1018-1029. https:// doi.org/10.1080/02508060.2014.992720

Thaler, T. (2016): Moving away from local-based flood risk policy in Austria. Regional Studies, Regional Science, 3, 329-336. https://doi.org/10. 1080/21681376.2016.1195282

Thaler, T. \& Levin-Keitel, M. (2016): Multi-level stakeholder engagement in flood risk management - A question of roles and power: Lessons from England. Environmental Science \& Policy, 55, 292-301. https://doi.org/10.1016/j.envsci. 2015.04.007

Thaler, T., Priest, S. \& Fuchs, S. (2016): Evolving inter-regional co-operation in flood risk management: Distances and types of partnership approaches in Austria. Regional Environmental Change, 16, 841-853. https://doi.org/10.1007/ s10113-015-0796-z

Thaler, T., Löschner, L. \& Hartmann, T. (2017): The introduction of catchment-wide co-operations: Scalar reconstructions and transformation in Austria in flood risk management. Land Use Policy, 68, 563-573. https://doi.org/10.1016/j. landusepol.2017.08.023

White, G. (1945): Human Adjustment to Floods: A Geographical Approach to the Flood Problem in the United States (= Geography Research Paper. Band 29). Chicago: Chicago University Press White, G., Kates, R. \& Burton, I. (2001): Knowing better and losing even more: the use of knowledge in hazards management. Environmental Hazards, 3-4, 81-92. https://doi.org/10. 1016/S1464-2867(01)00021-3 